\title{
AN OPTIMAL ERROR BOUND FOR A FINITE ELEMENT APPROXIMATION OF A MODEL FOR PHASE SEPARATION OF A MULTI-COMPONENT ALLOY WITH NON-SMOOTH FREE ENERGY
}

\author{
John W. BARRETT ${ }^{1}$ AND JAMes F. BloweY ${ }^{2}$
}

\begin{abstract}
Using the approach in [5] for analysing time discretization error and assuming more regularity on the initial data, we improve on the error bound derived in [2] for a fully practical piecewise linear finite element approximation with a backward Euler time discretization of a model for phase separation of a multi-component alloy with non-smooth free energy.
\end{abstract}

AMS Subject Classification. 65M60, 65M15, 35K55, 35K35.

Received: March 6, 1998.

\section{INTRODUCTION}

In [2], we proved an error bound for a fully practical finite element approximation of the following "deep quench" Cahn-Hilliard model:

(P) Find $\{\boldsymbol{u}(\cdot, t), \boldsymbol{w}(\cdot, t), \xi(\cdot, t)\} \in \boldsymbol{K} \times \boldsymbol{Y} \times L^{2}(\Omega)$ such that $\boldsymbol{u}(\cdot, 0)=\boldsymbol{u}^{0}(\cdot)$ and for a.e. $t \in(0, T)$

$$
\begin{array}{clrl}
\left\langle\frac{\partial \boldsymbol{u}}{\partial t}, \boldsymbol{\eta}\right\rangle+(L \nabla \boldsymbol{w}, \nabla \boldsymbol{\eta}) & =0 & & \forall \boldsymbol{\eta} \in \boldsymbol{H}^{1}(\Omega), \\
\gamma(\nabla \boldsymbol{u}, \nabla(\boldsymbol{\eta}-\boldsymbol{u}))-\left(\left(I-\mathbf{1} \sum\right) A \boldsymbol{u}, \boldsymbol{\eta}-\boldsymbol{u}\right) \geqslant(\boldsymbol{w}+\xi \mathbf{1}, \boldsymbol{\eta}-\boldsymbol{u}) & & \forall \boldsymbol{\eta} \in \boldsymbol{K}
\end{array}
$$

where

$$
\begin{array}{ll}
\boldsymbol{Y}:=\left\{\boldsymbol{\eta} \in \boldsymbol{H}^{1}(\Omega): \sum \boldsymbol{\eta}(\boldsymbol{x})=0\right. & \text { for a.e. } \boldsymbol{x} \in \Omega\} \\
\boldsymbol{K}:=\left\{\boldsymbol{\eta} \in \boldsymbol{H}^{1}(\Omega): \boldsymbol{\eta}(\boldsymbol{x}) \geqslant \mathbf{0}\right. & \text { for a.e. } \boldsymbol{x} \in \Omega\}
\end{array}
$$

Here $\Omega$ is a bounded domain in $\mathbb{R}^{d}(d \leqslant 3)$ with a Lipschitz boundary $\partial \Omega$.

In the above $\{\boldsymbol{u}\}_{n}$ is the fractional concentration of the $n^{\text {th }}$ component of the alloy, and so the following assumptions are made on the initial data

$$
\text { (a) } \boldsymbol{u}^{0}(\boldsymbol{x}) \geqslant \mathbf{0} \quad \text { and } \quad \text { (b) } N \sum \boldsymbol{u}^{0}(\boldsymbol{x})=1 \quad \forall \boldsymbol{x} \in \Omega \text {. }
$$

Keywords and phrases. Finite elements, error analysis, Cahn-Hilliard, phase separation.

${ }^{1}$ Department of Mathematics, Imperial College, London, SW7 2BZ, United Kingdom. e-mail: j.barrett@ic.ac.uk

2 Department of Mathematical Sciences, South Road, Durham, DH1 3LE, United Kingdom. 
In $(1.1 \mathrm{a}, \mathrm{b}) \gamma$ is a positive constant and $A$ and $L$ are symmetric constant $N \times N$ matrices. It is further assumed that

$$
L \text { has a one dimensional kernel such that } L \mathbf{1}=\mathbf{0} \text { and }
$$

$$
L \text { is positive semi-definite. }
$$

From physical considerations $A$ must have at least one positive eigenvalue, and the analysis simplifies if this were not the case. Let $\lambda_{A \max }$ be the largest positive eigenvalue of $A$.

We define $\mathbf{1} \in \mathbb{R}^{N}$ by $\{\mathbf{1}\}_{n}:=1$ for $n=1 \rightarrow N$. Here and throughout we write $\zeta_{n}$ for the $n^{\text {th }}$ component of $\zeta \in \mathbb{R}^{N}$ and set

$$
\sum \zeta:=\frac{1}{N} \sum_{n=1}^{N} \zeta_{n}
$$

For later purposes, we introduce for any $\mu \in \mathbb{R}$

$$
\mathcal{M}(\mu):=\left\{\boldsymbol{\xi} \in \mathbb{R}^{N}: N \sum \boldsymbol{\xi}=\mu\right\}
$$

Finally, we introduce

$$
f \boldsymbol{\eta}:=\frac{1}{|\Omega|} \int_{\Omega} \boldsymbol{\eta}(\boldsymbol{x}) \mathrm{d} \boldsymbol{x} \quad \forall \boldsymbol{\eta} \in \boldsymbol{L}^{2}(\Omega) .
$$

The system $(\mathrm{P})$ models the isothermal phase separation of a multi-component ideal mixture with $N \geqslant 2$ components in the deep quench limit, see [2] and the references cited therein. The well-posedness of (P), see Theorem 2.2 in [2], is proved under the following assumptions on the initial data $\boldsymbol{u}^{0}$ :

(D1) $\boldsymbol{u}^{0} \in \boldsymbol{H}^{1}(\Omega)$ such that (1.3) holds and $f \boldsymbol{u}^{0}>\delta \mathbf{1}$ for some $\delta \in(0,1 / N)$.

We note that the integral constraint above only excludes the degenerate case when one or more components of $\boldsymbol{u}$ are not present, in which case the system can be modelled with a smaller value of $N$.

The finite element approximation of $(\mathrm{P})$ was studied in [2] under the following assumptions:

(A1) Let $\Omega$ be convex polyhedral and $\mathcal{T}^{h}$ be a regular partitioning of $\Omega$ into disjoint open simplices $\kappa$ with $h_{\kappa}:=\operatorname{diam}(\kappa)$ and $h:=\max _{\kappa \in \mathcal{T} h} h_{\kappa}$, so that $\bar{\Omega} \equiv \cup_{\kappa \in \mathcal{T} h} \bar{\kappa}$.

In this paper we strengthen these assumptions to

(A2) In addition to (A1) let $\mathcal{T}^{h}$ be a quasi-uniform partitioning of $\Omega$.

Associated with $\mathcal{T}^{h}$ is the continuous piecewise linear finite element space

$$
S^{h}:=\left\{\chi \in C(\bar{\Omega}):\left.\chi\right|_{\tau} \text { is linear } \forall \tau \in \mathcal{T}^{h}\right\} \subset H^{1}(\Omega) .
$$

We extend these definitions to vector functions, i.e. $\chi \in \boldsymbol{S}^{h} \Rightarrow \chi_{n} \in S^{h}, n=1 \rightarrow N$.

Let $\pi^{h}: C(\bar{\Omega}) \rightarrow S^{h}$ be the interpolation operator such that $\pi^{h} \eta\left(\boldsymbol{x}_{m}\right)=\eta\left(\boldsymbol{x}_{m}\right)(m=1 \rightarrow M)$, where $\left\{\boldsymbol{x}_{m}\right\}_{m=1}^{M}$ is the set of nodes of $\mathcal{T}^{h}$. Throughout $(\cdot, \cdot)$ denotes the standard $L^{2}$ inner product over $\Omega$, naturally extended to vector and matrix functions, e.g. for $I \times J$ matrices $C(\boldsymbol{x})$ and $D(\boldsymbol{x})$, with entries in $L^{2}(\Omega)$

$$
(C, D):=\sum_{i=1}^{I} \sum_{j=1}^{J}\left(C_{i j}, D_{i j}\right):=\sum_{i=1}^{I} \sum_{j=1}^{J} \int_{\Omega} C_{i j}(\boldsymbol{x}) D_{i j}(\boldsymbol{x}) \mathrm{d} \boldsymbol{x} .
$$


Also $\langle\cdot, \cdot\rangle$ denotes the duality pairing between $\left(H^{1}(\Omega)\right)^{\prime}$ and $H^{1}(\Omega)$, which is extended to vector functions in the standard way. We now introduce the corresponding approximations of $(1.2 \mathrm{a}, \mathrm{b})$ :

$$
\begin{array}{ll}
\boldsymbol{Y}^{h}:=\left\{\boldsymbol{\chi} \in \boldsymbol{S}^{h}: \mathcal{\sum} \boldsymbol{\chi}\left(\boldsymbol{x}_{m}\right)=0,\right. & m=1 \rightarrow M\} \\
\boldsymbol{K}^{h}:=\left\{\boldsymbol{\chi} \in \boldsymbol{S}^{h}: \boldsymbol{\chi}\left(\boldsymbol{x}_{m}\right) \geqslant \mathbf{0},\right. & m=1 \rightarrow M\}
\end{array}
$$

A discrete semi-inner product on $C(\bar{\Omega})$ is then defined by

$$
\left(\eta_{1}, \eta_{2}\right)^{h}:=\int_{\Omega} \pi^{h}\left(\eta_{1}(\boldsymbol{x}) \eta_{2}(\boldsymbol{x})\right) \mathrm{d} \boldsymbol{x} \equiv \sum_{m=1}^{M} \beta_{m} \eta_{1}\left(\boldsymbol{x}_{m}\right) \eta_{2}\left(\boldsymbol{x}_{m}\right)
$$

where $\beta_{m}>0$. Once again, this is naturally extended to vector and matrix functions as in (1.6).

Given $K$, a positive integer, let $\Delta t:=T / K$ denote the time step and $t_{k}:=k \Delta t, k=1 \rightarrow K$; Barrett and Blowey [2] considered the following fully practical piecewise linear finite element approximation, based on a backward Euler time discretization, of $(\mathrm{P})$ :

$\left(\mathbf{P}^{h, \Delta t}\right)$ For $k=1 \rightarrow K$ find $\left\{\boldsymbol{U}^{k}, \boldsymbol{W}^{k}, \Xi^{k}\right\} \in \boldsymbol{K}^{h} \times \boldsymbol{Y}^{h} \times S^{h}$ such that

$$
\begin{array}{r}
\left(\frac{\boldsymbol{U}^{k}-\boldsymbol{U}^{k-1}}{\Delta t}, \boldsymbol{\chi}\right)^{h}+\left(L \nabla \boldsymbol{W}^{k}, \nabla \boldsymbol{\chi}\right)=0 \quad \forall \boldsymbol{\chi} \in \boldsymbol{S}^{h}, \\
\gamma\left(\nabla \boldsymbol{U}^{k}, \nabla\left(\boldsymbol{\chi}-\boldsymbol{U}^{k}\right)\right)-\left((I-\mathbf{1} \Sigma) A \boldsymbol{U}^{k}+\boldsymbol{W}^{k}+\Xi^{k} \mathbf{1}, \boldsymbol{\chi}-\boldsymbol{U}^{k}\right)^{h} \geqslant 0 \quad \forall \boldsymbol{\chi} \in \boldsymbol{K}^{h}
\end{array}
$$

where $\boldsymbol{U}^{0}=\boldsymbol{Q}_{i}^{h} \boldsymbol{u}^{0}$ for $i=1$ or 2 . Here

(i) $\boldsymbol{Q}_{1}^{h}: \boldsymbol{L}^{2}(\Omega) \rightarrow \boldsymbol{S}^{h}$ is such that $\left\{\boldsymbol{Q}_{1}^{h} \boldsymbol{\eta}\right\}_{n} \equiv Q_{1}^{h} \eta_{n}$ and $Q_{1}^{h}: L^{2}(\Omega) \rightarrow S^{h}$ is defined by

$$
\left(Q_{1}^{h} \eta, \chi\right)^{h}=(\eta, \chi) \quad \forall \chi \in S^{h}
$$

(ii) $\boldsymbol{Q}_{2}^{h}: \boldsymbol{H}^{1}(\Omega) \rightarrow \boldsymbol{S}^{h}$ is such that $\left\{\boldsymbol{Q}_{2}^{h} \boldsymbol{\eta}\right\}_{n} \equiv Q_{2}^{h} \eta_{n}$ and $Q_{2}^{h}: H^{1}(\Omega) \rightarrow S^{h}$ is defined by

$$
\gamma\left(\nabla\left(I-Q_{2}^{h}\right) \eta, \nabla \chi\right)+\left(\left(I-Q_{2}^{h}\right) \eta, \chi\right)=0 \quad \forall \chi \in S^{h}
$$

Let the assumptions (D1) and (A1) hold. Let $\boldsymbol{U}^{0}=\boldsymbol{Q}_{1}^{h} \boldsymbol{u}^{0}$. Then for all $h>0$ and all $\Delta t<4 \gamma /\left(\lambda_{A \max }^{2}\|L\|\right)$, Barrett and Blowey [2] proved the well-posedness of $\left(\mathrm{P}^{h, \Delta t}\right)$ on assuming that $\left\|\boldsymbol{U}^{0}\right\|_{1} \leqslant C$. Moreover, they proved that

$$
\|\boldsymbol{u}-\hat{\boldsymbol{U}}\|_{L^{2}\left(0, T ; \boldsymbol{H}^{1}(\Omega)\right)}^{2}+\|\boldsymbol{u}-\boldsymbol{U}\|_{L^{\infty}\left(0, T ;\left(\boldsymbol{H}^{1}(\Omega)\right)^{\prime}\right)}^{2} \leqslant C\left[h^{2}+\Delta t+\frac{h^{4}}{\Delta t}\right]
$$

Here we have adopted the notation: for $k \geq 1$

and

$$
\boldsymbol{U}(\cdot, t):=\frac{t-t_{k-1}}{\Delta t} \boldsymbol{U}^{k}(\cdot)+\frac{t_{k}-t}{\Delta t} \boldsymbol{U}^{k-1}(\cdot) \quad t \in\left[t_{k-1}, t_{k}\right]
$$

$$
\hat{\boldsymbol{U}}(\cdot, t):=\boldsymbol{U}^{k}(\cdot) \quad t \in\left(t_{k-1}, t_{k}\right]
$$

In the above and throughout the paper, $\|\cdot\|$ operating on matrices is that induced by the Euclidean vector norm, i.e. the spectral radius for symmetric matrices. We note that the assumption $\left\|U^{0}\right\|_{1} \leqslant C$ holds under 
the stronger mesh assumptions (A2). It follows immediately from (1.12) with the choice of $\Delta t \equiv C_{1} h^{2}<$ $4 \gamma /\left(\lambda_{A \max }^{2}\|L\|\right)$ that

$$
\|\boldsymbol{u}-\hat{\boldsymbol{U}}\|_{L^{2}\left(0, T ; \boldsymbol{H}^{1}(\Omega)\right)}^{2}+\|\boldsymbol{u}-\boldsymbol{U}\|_{L^{\infty}\left(0, T ;\left(\boldsymbol{H}^{1}(\Omega)\right)^{\prime}\right)}^{2} \leqslant C h^{2} .
$$

It is the purpose of this paper to improve on the error bound (1.12) using the approach developed by Rulla [5] for proving an optimal time discretization error for the backward Euler method applied to "subgradient flows" without requiring bounds on the second order time derivatives, which do not exist for the variational inequality system $(\mathrm{P})$. This approach does require the following stronger assumptions on the initial data:

(D2) $\boldsymbol{u}^{0} \in \boldsymbol{H}^{3}(\Omega)$ such that (1.3b) holds, $\partial \boldsymbol{u}^{0} / \partial \boldsymbol{\nu}=0$ on $\partial \Omega$, where $\boldsymbol{\nu}$ is normal to $\partial \Omega$, and $\boldsymbol{u}^{0}(\boldsymbol{x}) \geqslant \delta \mathbf{1}, \forall \boldsymbol{x} \in$ $\bar{\Omega}$ for some $\delta \in(0,1 / N]$.

With (D1, A1) replaced by (D2, A2), $\boldsymbol{U}^{0}=\boldsymbol{Q}_{2}^{h} \boldsymbol{u}^{0}$ and the restriction $h \leqslant h_{0}$; we prove in this paper that the term " $\Delta t+h^{4} / \Delta t$ " on the right-hand side of (1.12) can be replaced by " $(\Delta t)^{2}$ ", yielding an optimal error bound. Hence the bound (1.14) can be achieved by choosing larger time steps; $\Delta t \equiv C_{2} h<4 \gamma /\left(\lambda_{A \max }^{2}\|L\|\right)$.

Remark 1.1. In the case $N=2$, assuming that $A_{11}=A_{22}, L_{11}=L_{22}=1 / 2$, defining $u:=u_{2}-u_{1}$, $w:=w_{2}-w_{1}$ and $\theta_{c}=A_{11}-A_{12}$ we obtain that $\{u, w\}$ satisfies the system

$$
\frac{\partial u}{\partial t}-\Delta w=0, \quad w \in-\gamma \Delta u-\theta_{c} u+\partial I_{[-1,1]}(u)
$$

where $\partial I_{[-1,1]}(\cdot)$ is the subdifferential of the indicator function of the set $[-1,1]$. This is the Cahn-Hilliard equation with an obstacle free energy. The corresponding finite element approximation of this problem has been studied by Blowey and Elliott [4]. Obviously the results in this paper are easily adapted to improve on the error bound derived there in an analogous way.

\section{Notation and auxiliary results}

We adopt the standard notation for Sobolev spaces, denoting the norm of $W^{m, p}(\Omega)(m \in \mathbb{N}, p \in[1, \infty])$ by $\|\cdot\|_{m, p}$ and the semi-norm by $|\cdot|_{m, p}$. We extend these norms and semi-norms in the natural way to the corresponding spaces of vector functions $\boldsymbol{W}^{m, p}(\Omega):=\left\{W^{m, p}(\Omega)\right\}^{N}$. For $p=2, W^{m, 2}(\Omega)$ will be denoted by $H^{m}(\Omega)$, with the associated norm and semi-norm written as, respectively, $\|\cdot\|_{m}$ and $|\cdot|_{m}$. Furthermore, we define $\boldsymbol{L}^{2}\left(\Omega_{T}\right):=L^{2}\left(0, T ; \boldsymbol{L}^{2}(\Omega)\right)$. For $\boldsymbol{\eta} \in \boldsymbol{H}^{1}(\Omega), \nabla \boldsymbol{\eta}$ denotes the $N \times d$ matrix with entries $\{\nabla \boldsymbol{\eta}\}_{i j}:=\partial \eta_{i} / \partial x_{j}$ and then $\partial \boldsymbol{\eta} / \partial \boldsymbol{\nu}:=(\nabla \boldsymbol{\eta}) \boldsymbol{\nu}$.

Below we recall some well-known results concerning $S^{h}$ under the assumptions (A2): The inverse inequality for $1 \leqslant p_{1} \leqslant p_{2} \leqslant \infty$ and $m=0$ or 1

$$
|\chi|_{m, p_{2}} \leqslant C h^{\frac{d\left(p_{1}-p_{2}\right)}{p_{1} p_{2}}}|\chi|_{m, p_{1}} \quad \forall \chi \in S^{h} .
$$

For $m=0$ or 1 and $p \geqslant 2$

$$
\begin{array}{rlrl}
|\chi|_{0} \leqslant|\chi|_{h}:=\left[(\chi, \chi)^{h}\right]^{\frac{1}{2}} & \leqslant(d+2)^{\frac{1}{2}}|\chi|_{0} & \forall \chi \in S^{h}, \\
\left|\left(\chi_{1}, \chi_{2}\right)-\left(\chi_{1}, \chi_{2}\right)^{h}\right| \leqslant C h^{1+m}\left\|\chi_{1}\right\|_{m}\left\|\chi_{2}\right\|_{1} & \forall \chi_{1}, \chi_{2} \in S^{h}, \\
\left|\left(I-\pi^{h}\right) \eta\right|_{m, p} \leqslant C h^{2-m-d\left(\frac{1}{2}-\frac{1}{p}\right)}|\eta|_{2} & \forall \eta \in H^{2}(\Omega), \\
\left|\left(I-Q_{2}^{h}\right) \eta\right|_{m} \leqslant C h^{2-m}|\eta|_{2} & \forall \eta \in H^{2}(\Omega), \\
\left|\left(I-Q_{2}^{h}\right) \eta\right|_{m, p} \leqslant C h^{2-m-d\left(\frac{1}{2}-\frac{1}{p}\right)}|\eta|_{2} & \forall \eta \in H^{2}(\Omega)
\end{array}
$$

where the last result follows immediately from $(1.16,1.19,1.20)$. 
Below we recall the following "inverse Laplacian" operators introduced in [1]:

(a) $\mathcal{G}: \mathcal{F} \rightarrow V$ is such that

$$
(\nabla \mathcal{G} v, \nabla \eta)=\langle v, \eta\rangle \quad \forall \eta \in H^{1}(\Omega)
$$

where $\mathcal{F}:=\left\{v \in\left(H^{1}(\Omega)\right)^{\prime}:\langle v, 1\rangle=0\right\}$ and $V:=\left\{v \in H^{1}(\Omega):(v, 1)=0\right\}$.

(b) $\mathcal{G}: \mathcal{F} \rightarrow \boldsymbol{V}$ is defined by $\{\mathcal{G} \boldsymbol{v}\}_{n}:=\mathcal{G} v_{n}$, where

$$
\mathcal{F}:=\left\{\boldsymbol{v}: v_{n} \in \mathcal{F}, n=1 \rightarrow N, \text { and } \sum \boldsymbol{v}=0\right\}
$$

and

$$
\boldsymbol{V}:=\left\{\boldsymbol{v}: v_{n} \in V, n=1 \rightarrow N, \text { and } \sum \boldsymbol{v}=0\right\} .
$$

(c) Noting (1.4a, 1.5), it follows that $\left.L_{\mathcal{M}(0)} \equiv L\right|_{\mathcal{M}(0)}$ is invertible. Hence we can define $\mathcal{G}_{L}: \mathcal{F} \rightarrow \boldsymbol{V}$ by $\mathcal{G}_{L} \equiv L_{\mathcal{M}(0)}^{-1} \mathcal{G} ;$ that is

$$
\left(L \nabla \mathcal{G}_{L} \boldsymbol{v}, \nabla \boldsymbol{\eta}\right)=\langle\boldsymbol{v}, \boldsymbol{\eta}\rangle \quad \forall \boldsymbol{\eta} \in \boldsymbol{H}^{1}(\Omega) .
$$

(d) $\mathcal{G}^{h}: \mathcal{F} \rightarrow V^{h}:=\left\{v^{h} \in S^{h}:\left(v^{h}, 1\right)=0\right\}$ is such that

$$
\left(\nabla \mathcal{G}^{h} v, \nabla \chi\right)=\langle v, \chi\rangle \quad \forall \chi \in S^{h} .
$$

(e) $\mathcal{G}^{h}: \mathcal{F} \rightarrow \boldsymbol{V}^{h}$ is defined by $\left\{\mathcal{G}^{h} \boldsymbol{v}\right\}_{n}:=\mathcal{G}^{h} v_{n}$, where

$$
\boldsymbol{V}^{h}:=\left\{\boldsymbol{v}^{h}: v_{n}^{h} \in V^{h}, n=1 \rightarrow N, \text { and } \sum \boldsymbol{v}^{h}=0\right\} \subset \boldsymbol{V} .
$$

(f) $\mathcal{G}_{L}^{h}: \mathcal{F} \rightarrow \boldsymbol{V}^{h}$ is such that $\mathcal{G}_{L}^{h} \equiv L_{\mathcal{M}(0)}^{-1} \mathcal{G}^{h}$; that is,

$$
\left(L \nabla \mathcal{G}_{L}^{h} \boldsymbol{v}, \nabla \boldsymbol{\chi}\right)=\langle\boldsymbol{v}, \boldsymbol{\chi}\rangle \quad \forall \boldsymbol{\chi} \in \boldsymbol{S}^{h} .
$$

(g) $\hat{\mathcal{G}}^{h}: \mathcal{F}^{c} \rightarrow V^{h}$ is defined by

$$
\left(\nabla \hat{\mathcal{G}}^{h} v, \nabla \chi\right)=(v, \chi)^{h} \quad \forall \chi \in S^{h},
$$

where $\mathcal{F}^{c}:=\left\{v \in C(\bar{\Omega}):(v, 1)^{h}=0\right\}$.

(h) $\hat{\mathcal{G}}^{h}: \mathcal{F}^{c} \rightarrow \boldsymbol{V}^{h}$ is defined by $\left\{\hat{\mathcal{G}}^{h} \boldsymbol{v}\right\}_{n}:=\hat{\mathcal{G}}^{h} v_{n}$, where

$$
\mathcal{F}^{c}:=\left\{\boldsymbol{v}: v_{n} \in \mathcal{F}^{c}, n=1 \rightarrow N \text {, and } \sum \boldsymbol{v}=0\right\} \supset \boldsymbol{V}^{h} .
$$

(i) $\hat{\mathcal{G}}_{L}^{h}: \mathcal{F}^{c} \rightarrow \boldsymbol{V}^{h}$ is defined by $\hat{\mathcal{G}}_{L}^{h} \equiv L_{\mathcal{M}(0)}^{-1} \hat{\mathcal{G}}^{h}$; that is,

$$
\left(L \nabla \hat{\mathcal{G}}_{L}^{h} \boldsymbol{v}, \nabla \boldsymbol{\chi}\right)=(\boldsymbol{v}, \boldsymbol{\chi})^{h} \quad \forall \boldsymbol{\chi} \in \boldsymbol{S}^{h} .
$$

On noting the Poincaré inequality

$$
|\eta|_{0} \leqslant C\left(|\eta|_{1}+|(\eta, 1)|\right) \quad \forall \eta \in H^{1}(\Omega),
$$


the well-posedness of $\mathcal{G}, \mathcal{G}, \mathcal{G}^{h}, \mathcal{G}^{h}$ follows. In addition, on noting (1.17) we deduce the well-posedness of $\hat{\mathcal{G}}^{h}$ and $\hat{\mathcal{G}}^{h}$. Finally, as $\left.L_{\mathcal{M}(0)} \equiv L\right|_{\mathcal{M}(0)}$ is invertible, or equivalently noting that

$$
\lambda_{L \min }|\boldsymbol{v}|_{1}^{2} \leqslant(L \nabla \boldsymbol{v}, \nabla \boldsymbol{v}) \quad \forall \boldsymbol{v} \in \boldsymbol{V}
$$

where $\lambda_{L \text { min }}$ is the smallest positive eigenvalue of $L$; yields the well-posedness of $\mathcal{G}_{L}, \mathcal{G}_{L}^{h}$ and $\hat{\mathcal{G}}_{L}^{h}$.

Noting (1.25) one can then define a norm on $\mathcal{F}$ by

$$
\|\boldsymbol{v}\|_{-1}:=\left|L^{1 / 2} \mathcal{G}_{L} \boldsymbol{v}\right|_{1} \equiv\left[\left(L \nabla \mathcal{G}_{L} \boldsymbol{v}, \nabla \mathcal{G}_{L} \boldsymbol{v}\right)\right]^{1 / 2} \equiv\left[\left\langle\boldsymbol{v}, \mathcal{G}_{L} \boldsymbol{v}\right\rangle\right]^{1 / 2} \quad \forall \boldsymbol{v} \in \mathcal{F}
$$

It follows from $(1.33,1.34)$ that

$$
\lambda_{L \min }\left|\mathcal{G}_{L} \boldsymbol{v}\right|_{1}^{2} \leqslant\|\boldsymbol{v}\|_{-1}^{2} \quad \forall \boldsymbol{v} \in \mathcal{F} .
$$

In addition it follows from $(1.25,1.34,1.35)$ that

$$
\lambda_{L \min }\|\boldsymbol{v}\|_{-1}^{2} \leqslant|\mathcal{G} \boldsymbol{v}|_{1}^{2} \leqslant\|L\|\|\boldsymbol{v}\|_{-1}^{2} \quad \forall \boldsymbol{v} \in \mathcal{F} .
$$

Hence $\|\cdot\|_{-1}$ is equivalent to the standard $\left(\boldsymbol{H}^{1}(\Omega)\right)^{\prime}$ norm on $\mathcal{F}$. Similarly, one can define norms on $\mathcal{F}$ and $\mathcal{F}^{c}$ by

$$
\|\boldsymbol{v}\|_{-h}:=\left|L^{1 / 2} \mathcal{G}_{L}^{h} \boldsymbol{v}\right|_{1} \equiv\left[\left(L \nabla \mathcal{G}_{L}^{h} \boldsymbol{v}, \nabla \mathcal{G}_{L}^{h} \boldsymbol{v}\right)\right]^{1 / 2} \equiv\left[\left\langle\boldsymbol{v}, \mathcal{G}_{L}^{h} \boldsymbol{v}\right\rangle\right]^{1 / 2} \quad \forall \boldsymbol{v} \in \mathcal{F}
$$

and

$$
\|\boldsymbol{v}\|_{-h, h}:=\left|L^{1 / 2} \hat{\mathcal{G}}_{L}^{h} \boldsymbol{v}\right|_{1} \equiv\left[\left(L \nabla \hat{\mathcal{G}}_{L}^{h} \boldsymbol{v}, \nabla \hat{\mathcal{G}}_{L}^{h} \boldsymbol{v}\right)\right]^{1 / 2} \equiv\left[\left(\boldsymbol{v}, \hat{\mathcal{G}}_{L}^{h} \boldsymbol{v}\right)^{h}\right]^{1 / 2} \quad \forall \boldsymbol{v} \in \mathcal{F}^{c},
$$

respectively. It follows from $(1.28,1.31,1.37,1.38)$ that for all $\boldsymbol{v}^{h} \in \boldsymbol{V}^{h}$ and for all $\alpha>0$

$$
\begin{aligned}
\left|\boldsymbol{v}^{h}\right|_{0}^{2} & \equiv\left(L^{1 / 2} \nabla \mathcal{G}_{L}^{h} \boldsymbol{v}^{h}, L^{1 / 2} \nabla \boldsymbol{v}^{h}\right) \leqslant \frac{1}{2 \alpha}\left\|\boldsymbol{v}^{h}\right\|_{-h}^{2}+\frac{\alpha}{2}\|L\|\left|\boldsymbol{v}^{h}\right|_{1}^{2}, \\
\left|\boldsymbol{v}^{h}\right|_{h}^{2} & \equiv\left(L^{1 / 2} \nabla \hat{\mathcal{G}}_{L}^{h} \boldsymbol{v}^{h}, L^{1 / 2} \nabla \boldsymbol{v}^{h}\right) \leqslant \frac{1}{2 \alpha}\left\|\boldsymbol{v}^{h}\right\|_{-h, h}^{2}+\frac{\alpha}{2}\|L\|\left|\boldsymbol{v}^{h}\right|_{1}^{2} .
\end{aligned}
$$

It is well-known that

$$
\left|\left(\mathcal{G}-\mathcal{G}^{h}\right) \eta\right|_{0} \leqslant C h^{2-m}\left|\mathcal{G}^{m} \eta\right|_{m}, \quad \forall \eta \in\left(H^{m}(\Omega)\right)^{\prime} \cap \mathcal{F}, \quad m=0 \text { or } 1 .
$$

Hence, it follows from $(1.25,1.28,1.36,1.40)$ that

$$
\begin{array}{r}
\left|\left(\mathcal{G}_{L}-\mathcal{G}_{L}^{h}\right) \boldsymbol{\eta}\right|_{0} \leqslant \lambda_{L \text { min }}^{-1}\left|\left(\mathcal{G}-\mathcal{G}^{h}\right) \boldsymbol{\eta}\right|_{0} \leqslant C h^{2-m}\left|\mathcal{G}^{m} \boldsymbol{\eta}\right|_{m} \leqslant C h^{2-m}\|\boldsymbol{\eta}\|_{-m} \\
\forall \boldsymbol{\eta} \in\left(\boldsymbol{H}^{m}(\Omega)\right)^{\prime} \cap \mathcal{F}, \quad m=0 \text { or } 1 .
\end{array}
$$

It is easily deduced from (1.18), e.g. see [4], that

$$
\left\|\left(\mathcal{G}^{h}-\hat{\mathcal{G}}^{h}\right) v^{h}\right\|_{1} \leqslant C h^{2}\left\|v^{h}\right\|_{1} \quad \forall v^{h} \in V^{h} .
$$

Hence it follows from $(1.28,1.31,1.42)$ that

$$
\left\|\left(\mathcal{G}_{L}^{h}-\hat{\mathcal{G}}_{L}^{h}\right) \boldsymbol{v}^{h}\right\|_{1} \leqslant \lambda_{L \min }^{-1}\left\|\left(\mathcal{G}^{h}-\hat{\mathcal{G}}^{h}\right) \boldsymbol{v}^{h}\right\|_{1} \leqslant C h^{2}\left\|\boldsymbol{v}^{h}\right\|_{1}, \quad \forall \boldsymbol{v}^{h} \in \boldsymbol{V}^{h}
$$


Next we note that

$$
C_{1} h^{2}\left|\boldsymbol{v}^{h}\right|_{1} \leqslant C_{2} h\left|\boldsymbol{v}^{h}\right|_{0} \leqslant\left\|\boldsymbol{v}^{h}\right\|_{-h} \leqslant\left\|\boldsymbol{v}^{h}\right\|_{-1} \leqslant C_{3}\left\|\boldsymbol{v}^{h}\right\|_{-h} \quad \forall \boldsymbol{v}^{h} \in \boldsymbol{V}^{h}
$$

The first inequality on the left is just an inverse inequality, recalling that the partitioning is quasi-uniform. The second follows from the first and (1.39a). The third follows from noting that $\left|L^{1 / 2} \mathcal{G}_{L}^{h} \boldsymbol{v}^{h}\right|_{1} \leqslant\left|L^{1 / 2} \mathcal{G}_{L} \boldsymbol{v}^{h}\right|_{1}$. The final inequality follows from noting (1.41) with $m=0$ and the second inequality above. Finally, we have an analogue of (1.44)

$$
h^{2}\left|\boldsymbol{v}^{h}\right|_{1} \leqslant C_{1} h\left|\boldsymbol{v}^{h}\right|_{h} \leqslant C_{2}\left\|\boldsymbol{v}^{h}\right\|_{-h, h} \leqslant C_{3}\left\|\boldsymbol{v}^{h}\right\|_{-h} \leqslant C_{4}\left\|\boldsymbol{v}^{h}\right\|_{-h, h} \quad \forall \boldsymbol{v}^{h} \in \boldsymbol{V}^{h} .
$$

The first inequality on the left is just an inverse inequality on noting (1.17). The second follows from the first and (1.39b). The third and fourth follow from (1.43) and noting the first two inequalities in (1.44) and (1.45), respectively.

\section{The continuous problem}

It is easily established, see [2] for details, that $(\mathrm{P})$ can be rewritten as:

Find $\{\boldsymbol{u}(\cdot, t), \boldsymbol{\lambda}(t), \xi(\cdot, t)\} \in \boldsymbol{K}_{\boldsymbol{m}} \times \mathcal{M}(0) \times L^{2}(\Omega)$ such that $\boldsymbol{u}(\cdot, 0)=\boldsymbol{u}^{0}(\cdot)$ and for a.e. $t \in(0, T)$

$$
\gamma(\nabla \boldsymbol{u}, \nabla(\boldsymbol{\eta}-\boldsymbol{u}))+\left(\mathcal{G}_{L} \frac{\partial \boldsymbol{u}}{\partial t}-\left(I-\mathbf{1} \sum\right) A \boldsymbol{u}-\boldsymbol{\lambda}-\xi \mathbf{1}, \boldsymbol{\eta}-\boldsymbol{u}\right) \geqslant 0 \quad \forall \boldsymbol{\eta} \in \boldsymbol{K}
$$

where

$$
\begin{aligned}
\tilde{\boldsymbol{K}} & :=\left\{\boldsymbol{\eta} \in \boldsymbol{K} \text { and } N \sum \boldsymbol{\eta}(\boldsymbol{x})=1 \text { for a.e. } \boldsymbol{x} \in \Omega\right\} \\
\boldsymbol{K}_{\boldsymbol{m}} & :=\left\{\boldsymbol{\eta} \in \tilde{\boldsymbol{K}} \text { and } f \boldsymbol{\eta}=\boldsymbol{m}:=f \boldsymbol{u}^{0}\right\} .
\end{aligned}
$$

In the above we have eliminated $\boldsymbol{w} \in \boldsymbol{Y}$ by noting from $(1.1 \mathrm{a}, 1.25,1.33,1.32)$ that

$$
\boldsymbol{w} \equiv-\mathcal{G}_{L} \frac{\partial \boldsymbol{u}}{\partial t}+\boldsymbol{\lambda}
$$

where $\boldsymbol{\lambda}(t) \in \mathcal{M}(0)$ can be viewed as an unknown Lagrange multiplier.

Theorem 2.1. Let the assumptions (D2) hold. Let $\Omega$ be convex polyhedral or $\partial \Omega \in C^{1,1}$. Then there exists a unique solution $\{\boldsymbol{u}(\cdot, t), \boldsymbol{\lambda}(t), \xi(\cdot, t)\}(\equiv\{\boldsymbol{u}(\cdot, t), \boldsymbol{w}(\cdot, t), \xi(\cdot, t)\})$ to $(P)$ such that the following stability bounds hold:

$$
\begin{aligned}
\|\boldsymbol{u}\|_{W^{1, \infty}\left(0, T ;\left(\boldsymbol{H}^{1}(\Omega)\right)^{\prime}\right)}+\|\boldsymbol{u}\|_{H^{1}\left(0, T ; \boldsymbol{H}^{1}(\Omega)\right)} & +\|\boldsymbol{u}\|_{L^{\infty}\left(0, T ; \boldsymbol{H}^{2}(\Omega)\right)}+\|\boldsymbol{\lambda}\|_{\boldsymbol{L}^{\infty}(0, T)} \\
& +\|\boldsymbol{w}\|_{L^{\infty}\left(0, T ; \boldsymbol{H}^{1}(\Omega)\right)}+\|\boldsymbol{w}\|_{L^{2}\left(0, T ; \boldsymbol{H}^{2}(\Omega)\right)}+\|\xi\|_{L^{\infty}\left(0, T ; \boldsymbol{L}^{2}(\Omega)\right)} \leqslant C .
\end{aligned}
$$

In addition we have for a.e. $t_{a}, t_{b}$ with $0<t_{a}<t_{b}<T$ that

$$
\left\|\frac{\partial \boldsymbol{u}}{\partial t}\left(\cdot, t_{b}\right)\right\|_{-1}^{2} \leqslant\left\|\frac{\partial \boldsymbol{u}}{\partial t}\left(\cdot, t_{a}\right)\right\|_{-1}^{2}+C\left(t_{b}-t_{a}\right) .
$$

Proof. Existence, uniqueness and the bounds (2.4) are proved in Theorem 2.1 of [3] for a concentration dependent mobility matrix $L$. We note that the bounds (2.4) hold for any $T>0$ for the present case of a constant mobility matrix $L$. 
For a.e. $t \in(\delta t, T)$ and for all $\delta t>0$, on choosing $\boldsymbol{\eta} \equiv \boldsymbol{u}(\cdot, t-\delta t) \in \boldsymbol{K}_{\boldsymbol{m}}$ in $(2.1)$ and $\boldsymbol{\eta} \equiv \boldsymbol{u}(\cdot, t) \in \boldsymbol{K}_{\boldsymbol{m}}$ in (2.1) at " $t=t-\delta t$ ", adding, using (1.25), a Young's inequality and (1.34) it follows that

$$
\begin{aligned}
\gamma|\boldsymbol{u}(\cdot, t)-\boldsymbol{u}(\cdot, t-\delta t)|_{1}^{2}+\frac{1}{2} \frac{\mathrm{d}}{\mathrm{d} t}\|\boldsymbol{u}(\cdot, t)-\boldsymbol{u}(\cdot, t-\delta t)\|_{-1}^{2} \leqslant & (A(\boldsymbol{u}(\cdot, t)-\boldsymbol{u}(\cdot, t-\delta t)), \boldsymbol{u}(\cdot, t)-\boldsymbol{u}(\cdot, t-\delta t)) \\
\leqslant & \frac{\gamma}{2}|\boldsymbol{u}(\cdot, t)-\boldsymbol{u}(\cdot, t-\delta t)|_{1}^{2} \\
& +C\left(\gamma, \lambda_{A \max }\right)\|\boldsymbol{u}(\cdot, t)-\boldsymbol{u}(\cdot, t-\delta t)\|_{-1}^{2} \cdot
\end{aligned}
$$

Integrating (2.6) over $\left(t_{a}, t_{b}\right) \subseteq(\delta t, T)$, dividing through by $(\delta t)^{2}$, taking the limit as $\delta t \searrow 0$ and noting (2.4) yields that

$$
\begin{aligned}
\gamma \int_{t_{a}}^{t_{b}}\left|\frac{\partial \boldsymbol{u}}{\partial t}\right|_{1}^{2} \mathrm{~d} t+\left\|\frac{\partial \boldsymbol{u}}{\partial t}\left(\cdot, t_{b}\right)\right\|_{-1}^{2} & \leqslant\left\|\frac{\partial \boldsymbol{u}}{\partial t}\left(\cdot, t_{a}\right)\right\|_{-1}^{2}+C\left(\gamma, \lambda_{A \max }\right) \int_{t_{a}}^{t_{b}}\left\|\frac{\partial \boldsymbol{u}}{\partial t}\right\|_{-1}^{2} \mathrm{~d} t \\
& \leqslant\left\|\frac{\partial \boldsymbol{u}}{\partial t}\left(\cdot, t_{a}\right)\right\|_{-1}^{2}+C\left(t_{b}-t_{a}\right) .
\end{aligned}
$$

Hence the desired result (2.5).

For later purposes, we note that $J: \boldsymbol{H}^{1}(\Omega) \rightarrow \mathbb{R}$ defined by

$$
J(\boldsymbol{\eta}):=\frac{\gamma}{2}|\boldsymbol{\eta}|_{1}^{2}-\frac{1}{2}(A \boldsymbol{\eta}, \boldsymbol{\eta}) \quad \forall \boldsymbol{\eta} \in \boldsymbol{H}^{1}(\Omega)
$$

is a Lyapunov functional for $(\mathrm{P})$. To see this, we fix $\delta t>0$ then it follows for a.e. $t \in(\delta t, T)$, on choosing $\boldsymbol{\eta} \equiv \boldsymbol{u}(\cdot, t-\delta t) \in \boldsymbol{K}_{\boldsymbol{m}}$ in $(2.1)$, that

$\gamma(\nabla \boldsymbol{u}(\cdot, t), \nabla(\boldsymbol{u}(\cdot, t)-\boldsymbol{u}(\cdot, t-\delta t)))+\delta t\left(\mathcal{G}_{L} \frac{\partial \boldsymbol{u}}{\partial t}(\cdot, t), \frac{\boldsymbol{u}(\cdot, t)-\boldsymbol{u}(\cdot, t-\delta t)}{\delta t}\right)-(A \boldsymbol{u}(\cdot, t), \boldsymbol{u}(\cdot, t)-\boldsymbol{u}(\cdot, t-\delta t)) \leqslant 0$.

Noting the identity

$$
-2(a-b) b=b^{2}-a^{2}+(a-b)^{2} \quad \forall a, b \in \mathbb{R},
$$

it follows from (2.9) and (2.8) that for a.e. $t \in(\delta t, T)$ and for all $\delta t>0$

$$
J(\boldsymbol{u}(\cdot, t))-J(\boldsymbol{u}(\cdot, t-\delta t))+\delta t\left(\mathcal{G}_{L} \frac{\partial \boldsymbol{u}}{\partial t}(\cdot, t), \frac{\boldsymbol{u}(\cdot, t)-\boldsymbol{u}(\cdot, t-\delta t)}{\delta t}\right) \leqslant \frac{(\delta t)^{2}}{2} \lambda_{A \max }\left|\frac{\boldsymbol{u}(\cdot, t)-\boldsymbol{u}(\cdot, t-\delta t)}{\delta t}\right|_{0}^{2}
$$

Dividing (2.11) by $\delta t$, integrating from $t=\delta t$ to $t_{k}$, taking the limit $\delta t \searrow 0$ and noting (2.4), (1.34) and (D2) yields for $k=1 \rightarrow K$ that

$$
J\left(\boldsymbol{u}\left(\cdot, t_{k}\right)\right)+\int_{0}^{t_{k}}\left\|\frac{\partial \boldsymbol{u}}{\partial t}(\cdot, t)\right\|_{-1}^{2} \mathrm{~d} t \leqslant J\left(\boldsymbol{u}^{0}\right) \leqslant C .
$$




\section{Finite element approximation}

Firstly, we note the following results concerning $\boldsymbol{Q}_{i}^{h}$. It follows immediately from $(1.10,1.11)$ and the assumptions (D1, A2) that for $i=1$ and 2

$$
\begin{gathered}
f \boldsymbol{Q}_{i}^{h} \boldsymbol{u}^{0}=f \boldsymbol{u}^{0}, \quad N \sum \boldsymbol{Q}_{i}^{h} \boldsymbol{u}^{0}(\boldsymbol{x})=1, \quad \forall \boldsymbol{x} \in \bar{\Omega}, \\
\text { and } \quad\left\|\boldsymbol{Q}_{i}^{h} \boldsymbol{u}^{0}\right\|_{1} \leqslant C\left\|\boldsymbol{u}^{0}\right\|_{1} \leqslant C .
\end{gathered}
$$

Under the same assumptions it follows that

$$
\left\|\boldsymbol{u}^{0}-\boldsymbol{Q}_{1}^{h} \boldsymbol{u}^{0}\right\|_{-1} \leqslant C h\left\|\boldsymbol{u}^{0}\right\|_{0} \leqslant C h \quad \text { and } \quad \boldsymbol{Q}_{1}^{h} \boldsymbol{u}^{0}(\boldsymbol{x}) \geqslant \mathbf{0} \quad \forall \boldsymbol{x} \in \bar{\Omega},
$$

see [1] for details. Under the assumptions (D2, A2) it follows from $(1.20,1.32,1.34,1.35)$ that

$$
\left\|\boldsymbol{u}^{0}-\boldsymbol{Q}_{2}^{h} \boldsymbol{u}^{0}\right\|_{-1}^{2} \leqslant C\left|\boldsymbol{u}^{0}-\boldsymbol{Q}_{2}^{h} \boldsymbol{u}^{0}\right|_{0}^{2} \leqslant C h^{4}\left|\boldsymbol{u}^{0}\right|_{2}^{2} \leqslant C h^{4}
$$

and in addition from (1.21) with $m=0$ and $p=\infty$ that for $h \leqslant h_{0}$

$$
\boldsymbol{Q}_{2}^{h} \boldsymbol{u}^{0}(\boldsymbol{x}) \geqslant \mathbf{0} \quad \forall \boldsymbol{x} \in \bar{\Omega}
$$

We now consider the finite element approximation $\left(\mathrm{P}^{h, \Delta t}\right)$, see $(1.9 \mathrm{a}, \mathrm{b})$, to $(\mathrm{P})$. Let

$$
\begin{aligned}
\tilde{\boldsymbol{K}}^{h} & :=\left\{\boldsymbol{\chi} \in \boldsymbol{K}^{h} \text { and } N \sum \boldsymbol{\chi}\left(\boldsymbol{x}_{m}\right)=1, m=1 \rightarrow M\right\}, \\
\boldsymbol{K}_{\boldsymbol{m}}^{h} & :=\left\{\boldsymbol{\chi} \in \tilde{\boldsymbol{K}}^{h} \text { and } f \boldsymbol{\chi}=\boldsymbol{m}:=f \boldsymbol{u}^{0}\right\} .
\end{aligned}
$$

Similarly to (2.1), on noting (3.1a), it is easily established, see [2] for details, that $\left(\mathrm{P}^{h, \Delta t}\right)$ can be rewritten as: For $k=1 \rightarrow K$, find $\left\{\boldsymbol{U}^{k}, \boldsymbol{\Lambda}^{k}, \Xi^{k}\right\} \in \boldsymbol{K}_{\boldsymbol{m}}^{h} \times \mathcal{M}(0) \times S^{h}$ such that

$$
\gamma\left(\nabla \boldsymbol{U}^{k}, \nabla\left(\boldsymbol{\chi}-\boldsymbol{U}^{k}\right)\right)+\left(\hat{\mathcal{G}}_{L}^{h}\left[\frac{\boldsymbol{U}^{k}-\boldsymbol{U}^{k-1}}{\Delta t}\right]-\left(I-\mathbf{1} \sum\right) A \boldsymbol{U}^{k}, \boldsymbol{\chi}-\boldsymbol{U}^{k}\right)^{h} \geqslant\left(\boldsymbol{\Lambda}^{k}+\Xi^{k} \mathbf{1}, \boldsymbol{\chi}-\boldsymbol{U}^{k}\right)^{h} \forall \boldsymbol{\chi} \in \boldsymbol{K}^{h},
$$

where $\boldsymbol{U}^{0}=\boldsymbol{Q}_{i}^{h} \boldsymbol{u}^{0}, i=1$ or 2 . In the above, similarly to (2.3), we have eliminated $\boldsymbol{W}^{k} \in \boldsymbol{Y}^{h}$ by noting from (1.9a, 1.31-1.33) that

$$
\boldsymbol{W}^{k} \equiv-\hat{\mathcal{G}}_{L}^{h}\left[\frac{\boldsymbol{U}^{k}-\boldsymbol{U}^{k-1}}{\Delta t}\right]+\boldsymbol{\Lambda}^{k} \quad k=1 \rightarrow K
$$

Theorem 3.1. Let the assumptions (D2) and (A2) hold. Let $\boldsymbol{U}^{0}=\boldsymbol{Q}_{2}^{h} \boldsymbol{u}^{0}$. Then for all $h \leqslant h_{0}$ and for all $\Delta t<4 \gamma /\left(\lambda_{\text {Amax }}^{2}\|L\|\right)$, there exists a solution $\left\{\boldsymbol{U}^{k}, \boldsymbol{\Lambda}^{k}, \Xi^{k}\right\}_{k=1}^{K}\left(\equiv\left\{\boldsymbol{U}^{k}, \boldsymbol{W}^{k}, \Xi^{k}\right\}_{k=1}^{K}\right)$ to $\left(P^{h, \Delta t}\right)$. Moreover $\left\{\boldsymbol{U}^{k}\right\}_{k=1}^{K}$ is unique and the following stability bounds hold

$$
\begin{aligned}
\|\boldsymbol{U}\|_{L^{\infty}\left(0, T ; \boldsymbol{H}^{1}(\Omega)\right)}+\|\boldsymbol{U}\|_{H^{1}\left(0, T ;\left(\boldsymbol{H}^{1}(\Omega)\right)^{\prime}\right)}+(\Delta t)^{-\frac{1}{2}}\|\boldsymbol{U}\|_{H^{1}\left(0, T ; \boldsymbol{H}^{1}(\Omega)\right)} \\
+\|\hat{\boldsymbol{W}}\|_{L^{2}\left(0, T ; \boldsymbol{H}^{1}(\Omega)\right)}+\|\hat{\boldsymbol{\Lambda}}\|_{L^{2}(0, T)}+\|\hat{\Xi}\|_{L^{2}\left(0, T ; L^{1}(\Omega)\right)} \leqslant C,
\end{aligned}
$$

where $\boldsymbol{U}$ and $\hat{\boldsymbol{U}}$ are defined as in $(1.13 a, b)$ with $\hat{\boldsymbol{W}}, \hat{\boldsymbol{\Lambda}}$ and $\hat{\Xi}$ being similarly defined. Furthermore we have that

$$
\|\boldsymbol{U}\|_{H^{1}\left(0, T ; \boldsymbol{H}^{1}(\Omega)\right)}+\|\boldsymbol{U}\|_{W^{1, \infty}\left(0, T ;\left(\boldsymbol{H}^{1}(\Omega)\right)^{\prime}\right)}+\|\hat{\boldsymbol{\Lambda}}\|_{L^{\infty}(0, T)} \leqslant C .
$$


Proof. Existence, uniqueness and the bounds (3.7) are proved in Theorem 3.1 of [2] with the assumption (D2) replaced by (D1) and the projection $\boldsymbol{Q}_{2}^{h}$ replaced by $\boldsymbol{Q}_{1}^{h}$ under no constraint on $h$. It is a simple matter to adapt these proofs to the projection $\boldsymbol{Q}_{2}^{h}$ with the mesh constraint on noting (3.1a, b) and (3.3a, b).

Therefore we need only prove (3.8). For the purposes of the analysis, it is convenient to introduce $\boldsymbol{U}^{-1}$ such that $\boldsymbol{U}^{0}-\boldsymbol{U}^{-1} \in \boldsymbol{V}^{h}$ and

$$
\gamma\left(\nabla \boldsymbol{U}^{0}, \nabla \boldsymbol{\chi}\right)-\left(A \boldsymbol{U}^{0}, \boldsymbol{\chi}\right)^{h}+\left(\hat{\mathcal{G}}_{L}^{h}\left[\frac{\boldsymbol{U}^{0}-\boldsymbol{U}^{-1}}{\Delta t}\right], \boldsymbol{\chi}\right)^{h}=0 \quad \forall \boldsymbol{\chi} \in \boldsymbol{V}^{h} .
$$

For $m \geqslant 1$, it follows from adding (3.5) with $k=m$ and $\boldsymbol{\chi} \equiv \boldsymbol{U}^{m-1}$ to (3.5) with $k=m-1$ and $\boldsymbol{\chi} \equiv \boldsymbol{U}^{m}[(3.9)$ if $m=1$ with $\boldsymbol{\chi} \equiv \boldsymbol{U}^{0}-\boldsymbol{U}^{1}$ ] that

$$
\begin{aligned}
\gamma\left|\nabla\left(\boldsymbol{U}^{m}-\boldsymbol{U}^{m-1}\right)\right|_{1}^{2} & -\left(A\left(\boldsymbol{U}^{m}-\boldsymbol{U}^{m-1}\right), \boldsymbol{U}^{m}-\boldsymbol{U}^{m-1}\right)^{h} \\
+ & \left(\hat{\mathcal{G}}_{L}^{h}\left[\frac{\boldsymbol{U}^{m}-\boldsymbol{U}^{m-1}}{\Delta t}-\frac{\boldsymbol{U}^{m-1}-\boldsymbol{U}^{m-2}}{\Delta t}\right], \boldsymbol{U}^{m}-\boldsymbol{U}^{m-1}\right)^{h} \leqslant 0 .
\end{aligned}
$$

Summing (3.10) for $m=1 \rightarrow k$, noting $(1.38,1.39 \mathrm{~b}, 2.10)$ yields for $k=1 \rightarrow K$ that

$$
\begin{aligned}
\gamma \Delta t \sum_{m=1}^{k}\left|\frac{\boldsymbol{U}^{m}-\boldsymbol{U}^{m-1}}{\Delta t}\right|_{1}^{2}+\frac{1}{2}\left\|\frac{\boldsymbol{U}^{k}-\boldsymbol{U}^{k-1}}{\Delta t}\right\|_{-h, h}^{2} & \leqslant \frac{1}{2}\left\|\frac{\boldsymbol{U}^{0}-\boldsymbol{U}^{-1}}{\Delta t}\right\|_{-h, h}^{2} \\
& +\Delta t \sum_{m=1}^{k}\left(A\left[\frac{\boldsymbol{U}^{m}-\boldsymbol{U}^{m-1}}{\Delta t}\right], \frac{\boldsymbol{U}^{m}-\boldsymbol{U}^{m-1}}{\Delta t}\right)^{h} \\
\leqslant & \left\|\frac{\boldsymbol{U}^{0}-\boldsymbol{U}^{-1}}{\Delta t}\right\|_{-h, h}^{2}+C(\gamma) \Delta t \sum_{m=1}^{k}\left\|\frac{\boldsymbol{U}^{m}-\boldsymbol{U}^{m-1}}{\Delta t}\right\|_{-h, h}^{2} .
\end{aligned}
$$

Choosing $\boldsymbol{\chi} \equiv \boldsymbol{U}^{0}-\boldsymbol{U}^{-1}$ in (3.9) and noting (1.11, 1.25, 1.34, 1.38), assumption (D2), (1.31, 1.44, 1.45, 3.1b) yields that

$$
\begin{aligned}
\left\|\frac{\boldsymbol{U}^{0}-\boldsymbol{U}^{-1}}{\Delta t}\right\|_{-h, h}^{2} & =-\gamma\left(\nabla \boldsymbol{U}^{0}, \nabla\left(\frac{\boldsymbol{U}^{0}-\boldsymbol{U}^{-1}}{\Delta t}\right)\right)+\left(A \boldsymbol{U}^{0}, \frac{\boldsymbol{U}^{0}-\boldsymbol{U}^{-1}}{\Delta t}\right)^{h} \\
& =\left(\gamma \Delta \boldsymbol{u}^{0}+\left(\boldsymbol{Q}_{2}^{h} \boldsymbol{u}^{0}-\boldsymbol{u}^{0}\right), \frac{\boldsymbol{U}^{0}-\boldsymbol{U}^{-1}}{\Delta t}\right)+\left(A \boldsymbol{U}^{0}, \frac{\boldsymbol{U}^{0}-\boldsymbol{U}^{-1}}{\Delta t}\right)^{h} \\
& \leqslant C\left\|\boldsymbol{u}^{0}\right\|_{3}^{2} \leqslant C .
\end{aligned}
$$

Hence combining $(3.11,3.12)$ and noting $(1.32,1.44,1.45,3.7)$ yields the first two bounds in (3.8).

The final bound in (3.8) follows from the second and recalling from (3.28-3.29) of [2] that

$$
\left\|\boldsymbol{\Lambda}^{k}\right\| \leqslant C\left[\left\|\frac{\boldsymbol{U}^{k}-\boldsymbol{U}^{k-1}}{\Delta t}\right\|_{-h, h}+1\right], \quad k=1 \rightarrow K .
$$

In Theorem 3.3 below we adapt the technique in Rulla [5] to improve on the temporal discretization error bound in [2] for the scheme $\left(\mathrm{P}^{h, \Delta t}\right)$. In the next lemma we bound a key term required in the proof of this theorem. 
Lemma 3.2. Let the assumptions of Theorem 3.1 hold. Then for $k=1 \rightarrow K$, we have that

$$
-\int_{0}^{t_{k}}\left\|\frac{\partial \boldsymbol{U}}{\partial t}\right\|_{-1}^{2} \mathrm{~d} t \leqslant J^{h}\left(\boldsymbol{U}^{k}\right)-J^{h}\left(\boldsymbol{Q}_{2}^{h} \boldsymbol{u}^{0}\right)+C\left[\Delta t+h^{2}\right]
$$

where $J^{h}: \boldsymbol{S}^{h} \rightarrow \mathbb{R}$ is defined by

$$
J^{h}(\chi):=\frac{\gamma}{2}|\chi|_{1}^{2}-\frac{1}{2}(A \chi, \chi)^{h} \quad \forall \chi \in S^{h} .
$$

Proof. Choosing $\boldsymbol{\chi}=\boldsymbol{U}^{m}$ in (3.5) with $k=m-1$ if $m \geqslant 2$ and $\boldsymbol{\chi}=\boldsymbol{U}^{1}-\boldsymbol{U}^{0}$ in (3.9), noting (2.10, 3.15) yields for $m=1 \rightarrow K$ that

$$
\begin{aligned}
J^{h}\left(\boldsymbol{U}^{m-1}\right)-J^{h}\left(\boldsymbol{U}^{m}\right)+\frac{\gamma}{2} \mid \boldsymbol{U}^{m} & -\left.\boldsymbol{U}^{m-1}\right|_{1} ^{2} \\
& \leqslant \Delta t\left(\hat{\mathcal{G}}_{L}^{h}\left[\frac{\boldsymbol{U}^{m-1}-\boldsymbol{U}^{m-2}}{\Delta t}\right], \frac{\boldsymbol{U}^{m}-\boldsymbol{U}^{m-1}}{\Delta t}\right)^{h}+\frac{1}{2} \lambda_{A \max }\left|\boldsymbol{U}^{m}-\boldsymbol{U}^{m-1}\right|_{h}^{2} .
\end{aligned}
$$

It follows from $(1.39 \mathrm{~b}, 1.44,1.45,3.8,3.16)$ that for $m=1 \rightarrow K$

$$
J^{h}\left(\boldsymbol{U}^{m-1}\right)-J^{h}\left(\boldsymbol{U}^{m}\right) \leqslant \Delta t\left(\hat{\mathcal{G}}_{L}^{h}\left[\frac{\boldsymbol{U}^{m-1}-\boldsymbol{U}^{m-2}}{\Delta t}\right], \frac{\boldsymbol{U}^{m}-\boldsymbol{U}^{m-1}}{\Delta t}\right)^{h}+C(\Delta t)^{2} .
$$

Noting $(1.38,2.10,3.17)$ we have for $m=1 \rightarrow K$ that

$$
\begin{aligned}
& -\Delta t\left\|\frac{\boldsymbol{U}^{m}-\boldsymbol{U}^{m-1}}{\Delta t}\right\|_{-h, h}^{2}+J^{h}\left(\boldsymbol{U}^{m-1}\right)-J^{h}\left(\boldsymbol{U}^{m}\right) \\
& \quad \leqslant \frac{1}{2} \Delta t\left[\left\|\frac{\boldsymbol{U}^{m-1}-\boldsymbol{U}^{m-2}}{\Delta t}\right\|_{-h, h}^{2}-\left\|\frac{\boldsymbol{U}^{m}-\boldsymbol{U}^{m-1}}{\Delta t}\right\|_{-h, h}^{2}\right]+C(\Delta t)^{2} .
\end{aligned}
$$

Summing (3.18) and noting (3.12), then yields for $k=1 \rightarrow K$ that

$$
-\int_{0}^{t_{k}}\left\|\frac{\partial \boldsymbol{U}}{\partial t}\right\|_{-h, h}^{2} \mathrm{~d} t-J^{h}\left(\boldsymbol{U}^{k}\right)+J^{h}\left(\boldsymbol{Q}_{2}^{h} \boldsymbol{u}^{0}\right) \leqslant \frac{1}{2} \Delta t\left\|\frac{\boldsymbol{U}^{0}-\boldsymbol{U}^{-1}}{\Delta t}\right\|_{-h, h}^{2}+C \Delta t \leqslant C \Delta t .
$$

The desired result (3.14) then follows from (3.19) on noting $(1.18,1.32,1.34,1.38,1.41,1.43,3.8)$.

Theorem 3.3. Let the assumptions (D2, A2) hold. Let $\boldsymbol{U}^{0}=\boldsymbol{Q}_{2}^{h} \boldsymbol{u}^{0}$. Then for all $h \leqslant h_{0}$ and for all $\Delta t<$ $4 \gamma /\left(\lambda_{\text {Amax }}^{2}\|L\|\right)$ we have that

$$
\|\boldsymbol{u}-\hat{\boldsymbol{U}}\|_{L^{2}\left(0, T ; \boldsymbol{H}^{1}(\Omega)\right)}^{2}+\|\boldsymbol{u}-\boldsymbol{U}\|_{L^{\infty}\left(0, T ;\left(\boldsymbol{H}^{1}(\Omega)\right)^{\prime}\right)}^{2}+\Delta t\|\boldsymbol{u}-\boldsymbol{U}\|_{H^{1}\left(0, T ;\left(\boldsymbol{H}^{1}(\Omega)\right)^{\prime}\right)}^{2} \leqslant C\left[h^{2}+(\Delta t)^{2}\right] .
$$

Proof. Using the notation (1.13a,b), (3.5) can be restated as:

Find $\boldsymbol{U} \in H^{1}\left(0, T ; \boldsymbol{K}_{\boldsymbol{m}}^{h}\right)$ such that $\boldsymbol{U}(\cdot, 0)=\boldsymbol{Q}_{2}^{h} \boldsymbol{u}^{0}(\cdot)$ and for a.e. $t \in(0, T)$

$$
\gamma(\nabla \hat{\boldsymbol{U}}, \nabla(\boldsymbol{\chi}-\hat{\boldsymbol{U}}))+\left(\hat{\mathcal{G}}_{L}^{h} \frac{\partial \boldsymbol{U}}{\partial t}-A \hat{\boldsymbol{U}}-\hat{\boldsymbol{\Lambda}}, \boldsymbol{\chi}-\hat{\boldsymbol{U}}\right)^{h} \geqslant 0 \quad \forall \boldsymbol{\chi} \in \tilde{\boldsymbol{K}}^{h} .
$$


We set $\boldsymbol{e}:=\boldsymbol{u}-\boldsymbol{U}, \hat{\boldsymbol{e}}:=\boldsymbol{u}-\hat{\boldsymbol{U}}, \boldsymbol{e}^{A}:=\boldsymbol{u}-\boldsymbol{\pi}^{h} \boldsymbol{u}, \boldsymbol{e}^{h}:=\boldsymbol{\pi}^{h} \boldsymbol{u}-\boldsymbol{U}$ and $\hat{\boldsymbol{e}}^{h}:=\boldsymbol{\pi}^{h} \boldsymbol{u}-\hat{\boldsymbol{U}}$. Note that $f \boldsymbol{e}=$ $f \boldsymbol{e}^{A}+f \boldsymbol{e}^{h}=\mathbf{0}, f \hat{\boldsymbol{e}}=f \boldsymbol{e}^{A}+f \hat{\boldsymbol{e}}^{h}=\mathbf{0}$ and $\sum \boldsymbol{e}^{A}=\sum \boldsymbol{e}^{h}=\sum \hat{\boldsymbol{e}}^{h}=0$. For a.e. $t \in(0, T)$ we have that

$$
|\hat{\boldsymbol{e}}|_{1}^{2} \leqslant\left(\nabla \hat{\boldsymbol{e}}, \nabla \hat{\boldsymbol{e}}^{h}\right)+|\hat{\boldsymbol{e}}|_{1}\left|\boldsymbol{e}^{A}\right|_{1}
$$

Introducing

$$
\mu^{\Delta t}(t):=\frac{t_{k}-t}{\Delta t} \quad t \in\left(t_{k-1}, t_{k}\right], \quad k=1 \rightarrow K
$$

we have that

$$
\boldsymbol{e}(\cdot, t)-\hat{\boldsymbol{e}}(\cdot, t)=\Delta t \mu^{\Delta t}(t) \frac{\partial \boldsymbol{U}}{\partial t}(\cdot, t), \quad t \in\left(t_{k-1}, t_{k}\right), \quad k=1 \rightarrow K
$$

It follows from $(2.10,3.24)$ that

$$
\begin{aligned}
\left(\mathcal{G}_{L} \frac{\partial \boldsymbol{e}}{\partial t}, \hat{\boldsymbol{e}}\right) & =\frac{1}{2} \frac{\mathrm{d}}{\mathrm{d} t}\|\boldsymbol{e}\|_{-1}^{2}-\Delta t \mu^{\Delta t}\left(\mathcal{G}_{L} \frac{\partial \boldsymbol{e}}{\partial t}, \frac{\partial \boldsymbol{U}}{\partial t}\right) \\
& =\frac{1}{2} \frac{\mathrm{d}}{\mathrm{d} t}\|\boldsymbol{e}\|_{-1}^{2}+\frac{\Delta t}{2} \mu^{\Delta t}\left[\left\|\frac{\partial \boldsymbol{e}}{\partial t}\right\|_{-1}^{2}+\left\|\frac{\partial \boldsymbol{U}}{\partial t}\right\|_{-1}^{2}-\left\|\frac{\partial \boldsymbol{u}}{\partial t}\right\|_{-1}^{2}\right]
\end{aligned}
$$

Next we note that

$$
\begin{aligned}
& \gamma\left(\nabla \hat{\boldsymbol{e}}, \nabla \hat{\boldsymbol{e}}^{h}\right)+\left(\mathcal{G}_{L} \frac{\partial \boldsymbol{e}}{\partial t}, \hat{\boldsymbol{e}}\right)=\left[\gamma\left(\nabla \boldsymbol{u}, \nabla \hat{\boldsymbol{e}}^{h}\right)+\left(\mathcal{G}_{L} \frac{\partial \boldsymbol{u}}{\partial t}, \hat{\boldsymbol{e}}\right)\right]-\left[\gamma\left(\nabla \hat{\boldsymbol{U}}, \nabla \hat{\boldsymbol{e}}^{h}\right)+\left(\hat{\mathcal{G}}_{L}^{h} \frac{\partial \boldsymbol{U}}{\partial t}, \hat{\boldsymbol{e}}^{h}\right)^{h}\right] \\
&+\left(\left(\hat{\mathcal{G}}_{L}^{h}-\mathcal{G}_{L}\right) \frac{\partial \boldsymbol{U}}{\partial t}, \hat{\boldsymbol{e}}^{h}\right)-\left(\mathcal{G}_{L} \frac{\partial \boldsymbol{U}}{\partial t}, \boldsymbol{e}^{A}\right)+\left[\left(\hat{\mathcal{G}}_{L}^{h} \frac{\partial \boldsymbol{U}}{\partial t}, \hat{\boldsymbol{e}}^{h}\right)^{h}-\left(\hat{\mathcal{G}}_{L}^{h} \frac{\partial \boldsymbol{U}}{\partial t}, \hat{\boldsymbol{e}}^{h}\right)\right]
\end{aligned}
$$

From (2.1), with $\boldsymbol{\eta} \equiv \hat{\boldsymbol{U}}$, and (2.4) it follows that

$$
\begin{aligned}
\gamma\left(\nabla \boldsymbol{u}, \nabla \hat{\boldsymbol{e}}^{h}\right)+\left(\mathcal{G}_{L} \frac{\partial \boldsymbol{u}}{\partial t}, \hat{\boldsymbol{e}}\right) & \leqslant\left(A \boldsymbol{u}+\boldsymbol{\lambda}, \hat{\boldsymbol{e}}^{h}\right)-\left[\gamma\left(\nabla \boldsymbol{u}, \nabla \boldsymbol{e}^{A}\right)-\left(A \boldsymbol{u}+\boldsymbol{\lambda}, \boldsymbol{e}^{A}\right)\right] \\
& =\left(A \boldsymbol{u}+\boldsymbol{\lambda}, \hat{\boldsymbol{e}}^{h}\right)-\left[\left(-\gamma \Delta \boldsymbol{u}-A \boldsymbol{u}-\boldsymbol{\lambda}, \boldsymbol{e}^{A}\right)\right]
\end{aligned}
$$

Choosing $\boldsymbol{\chi} \equiv \boldsymbol{\pi}^{h} \boldsymbol{u}$ in (3.21) yields that

$$
\gamma\left(\nabla \hat{\boldsymbol{U}}, \nabla \hat{\boldsymbol{e}}^{h}\right)+\left(\hat{\mathcal{G}}_{L}^{h} \frac{\partial \boldsymbol{U}}{\partial t}, \hat{\boldsymbol{e}}^{h}\right)^{h} \geqslant\left(A \hat{\boldsymbol{U}}+\hat{\boldsymbol{\Lambda}}, \hat{\boldsymbol{e}}^{h}\right)^{h}
$$


Combining $(3.22,3.25-3.28)$ and noting $(1.32,1.34,1.35,1.41,1.43)$, a Young's inequality, $(1.18,3.24)$ and that $(\boldsymbol{\lambda}-\hat{\Lambda}, \hat{e})=0$ yields

$$
\begin{aligned}
& \gamma|\hat{\boldsymbol{e}}|_{1}^{2}+\frac{1}{2} \frac{\mathrm{d}}{\mathrm{d} t}\|\boldsymbol{e}\|_{-1}^{2}+\frac{\Delta t}{2} \mu^{\Delta t}\left\|\frac{\partial \boldsymbol{e}}{\partial t}\right\|_{-1}^{2} \leqslant C\left[\|\boldsymbol{u}\|_{2}+\left\|\frac{\partial \boldsymbol{U}}{\partial t}\right\|_{-1}+\|\boldsymbol{\lambda}\|\right]\left|\boldsymbol{e}^{A}\right|_{0}+\left|\left(\mathcal{G}_{L}-\hat{\mathcal{G}}_{L}^{h}\right) \frac{\partial \boldsymbol{U}}{\partial t}\right|_{0}\left|\hat{\boldsymbol{e}}^{h}\right|_{0}+\left(A \hat{\boldsymbol{e}}, \hat{\boldsymbol{e}}^{h}\right) \\
& +\left[\left(A \hat{\boldsymbol{U}}, \hat{\boldsymbol{e}}^{h}\right)-\left(A \hat{\boldsymbol{U}}, \hat{\boldsymbol{e}}^{h}\right)^{h}\right]-\left(\boldsymbol{\lambda}-\hat{\boldsymbol{\Lambda}}, \boldsymbol{e}^{A}\right)+C h^{2}\left\|\hat{\mathcal{G}}_{L}^{h} \frac{\partial \boldsymbol{U}}{\partial t}\right\|_{1}\left\|\hat{\boldsymbol{e}}^{h}\right\|_{1} \\
& +\frac{\Delta t}{2} \mu^{\Delta t}\left[\left\|\frac{\partial \boldsymbol{u}}{\partial t}\right\|_{-1}^{2}-\left\|\frac{\partial \boldsymbol{U}}{\partial t}\right\|_{-1}^{2}\right]+\gamma|\hat{\boldsymbol{e}}|_{1}\left|\boldsymbol{e}^{A}\right|_{1} \\
& \leqslant \frac{1}{2} \gamma|\hat{\boldsymbol{e}}|_{1}^{2}+C\left[\|\boldsymbol{u}\|_{2}+\left\|\frac{\partial \boldsymbol{U}}{\partial t}\right\|_{-1}+\|\boldsymbol{\lambda}\|+|\hat{\boldsymbol{U}}|_{0}+\|\hat{\boldsymbol{\Lambda}}\|\right]\left|\boldsymbol{e}^{A}\right|_{0} \\
& +C h^{2}\left[\left\|\frac{\partial \boldsymbol{U}}{\partial t}\right\|_{1}+\|\hat{\boldsymbol{U}}\|_{1}\right]\left\|\hat{\boldsymbol{e}}^{h}\right\|_{1}+\frac{\Delta t}{2} \mu^{\Delta t}\left[\left\|\frac{\partial \boldsymbol{u}}{\partial t}\right\|_{-1}^{2}-\left\|\frac{\partial \boldsymbol{U}}{\partial t}\right\|_{-1}^{2}\right] \\
& +C\left[\|\boldsymbol{e}\|_{-1}^{2}+(\Delta t)^{2}\left\|\frac{\partial \boldsymbol{U}}{\partial t}\right\|_{-1}^{2}+\left|\boldsymbol{e}^{A}\right|_{1}^{2}\right] \text {. }
\end{aligned}
$$

Integrating (3.29), and noting that $\boldsymbol{e}(\cdot, 0)=\left(I-\boldsymbol{Q}_{2}^{h}\right) \boldsymbol{u}^{0}(\cdot), \partial \boldsymbol{U} / \partial t$ is constant over $\left(t_{k-1}, t_{k}\right),(1.19,2.4,3.3 \mathrm{a}, 3.7)$ yields that for $k=1 \rightarrow K$

$$
\begin{aligned}
\left\|\boldsymbol{e}\left(\cdot, t_{k}\right)\right\|_{-1}^{2}+\int_{0}^{t_{k}}[ & \left.\gamma|\hat{\boldsymbol{e}}|_{1}^{2}+\Delta t \mu^{\Delta t}\left\|\frac{\partial \boldsymbol{e}}{\partial t}\right\|_{-1}^{2}\right] \mathrm{d} t \\
\leqslant & \left\|\left(I-\boldsymbol{Q}_{2}^{h}\right) \boldsymbol{u}^{0}\right\|_{-1}^{2}+\int_{0}^{t_{k}}\left\{C\|\boldsymbol{e}\|_{-1}^{2}+\Delta t\left[\mu^{\Delta t}\left\|\frac{\partial \boldsymbol{u}}{\partial t}\right\|_{-1}^{2}-\frac{1}{2}\left\|\frac{\partial \boldsymbol{U}}{\partial t}\right\|_{-1}^{2}\right]\right\} \mathrm{d} t \\
& +C\left[\left\|\boldsymbol{e}^{A}\right\|_{L^{2}\left(0, T ; \boldsymbol{H}^{1}(\Omega)\right)}^{2}+(\Delta t)^{2}\left\|\frac{\partial \boldsymbol{U}}{\partial t}\right\|_{L^{2}\left(0, T ;\left(\boldsymbol{H}^{1}(\Omega)\right)^{\prime}\right)}^{2}\right] \\
& +C h^{2}\left[\left\|\frac{\partial \boldsymbol{U}}{\partial t}\right\|_{L^{2}\left(0, T ; \boldsymbol{H}^{1}(\Omega)\right)}+\|\hat{\boldsymbol{U}}\|_{L^{2}\left(0, T ; \boldsymbol{H}^{1}(\Omega)\right)}\right]\left\|\hat{\boldsymbol{e}}^{h}\right\|_{L^{2}\left(0, T ; \boldsymbol{H}^{1}(\Omega)\right)} \\
& +C\left[\|\boldsymbol{u}\|_{L^{2}\left(0, T ; \boldsymbol{H}^{2}(\Omega)\right)}+\left\|\frac{\partial \boldsymbol{U}}{\partial t}\right\|_{L^{2}\left(0, T ;\left(\boldsymbol{H}^{1}(\Omega)\right)^{\prime}\right)}+\|\boldsymbol{\lambda}\|_{\boldsymbol{L}^{2}(0, T)}+\|\hat{\boldsymbol{U}}\|_{\boldsymbol{L}^{2}\left(\Omega_{T}\right)}\right. \\
& \left.+\|\hat{\boldsymbol{\Lambda}}\|_{\boldsymbol{L}^{2}(0, T)}\right]\left\|\boldsymbol{e}^{A}\right\|_{\boldsymbol{L}^{2}\left(\Omega_{T}\right)} \\
\leqslant & \int_{0}^{t_{k}}\left\{C\|\boldsymbol{e}\|_{-1}^{2}+\Delta t\left[\mu^{\Delta t}\left\|\frac{\partial \boldsymbol{u}}{\partial t}\right\|_{-1}^{2}-\frac{1}{2}\left\|\frac{\partial \boldsymbol{U}}{\partial t}\right\|_{-1}^{2}\right]\right\} \mathrm{d} t+C\left[h^{2}+(\Delta t)^{2}\right] .
\end{aligned}
$$

Setting $\overline{\boldsymbol{u}}(\cdot, t):=\boldsymbol{u}(\cdot, t+\Delta t / 2)$ and defining $\overline{\boldsymbol{e}}:=\overline{\boldsymbol{u}}-\boldsymbol{U}$, it follows in an analogous manner to (3.30) that for $k=1 \rightarrow K$

$$
\begin{aligned}
\left\|\overline{\boldsymbol{e}}\left(\cdot, t_{k}\right)\right\|_{-1}^{2}+\Delta t \int_{0}^{t_{k}} \mu^{\Delta t}\left\|\frac{\partial \overline{\boldsymbol{e}}}{\partial t}\right\|_{-1}^{2} \mathrm{~d} t \leqslant & \|\overline{\boldsymbol{e}}(\cdot, 0)\|_{-1}^{2}+C\left[h^{2}+(\Delta t)^{2}\right] \\
& +\int_{0}^{t_{k}}\left\{C\|\overline{\boldsymbol{e}}\|_{-1}^{2}+\Delta t\left[\mu^{\Delta t}\left\|\frac{\partial \overline{\boldsymbol{u}}}{\partial t}\right\|_{-1}^{2}-\frac{1}{2}\left\|\frac{\partial \boldsymbol{U}}{\partial t}\right\|_{-1}^{2}\right]\right\} \mathrm{d} t .
\end{aligned}
$$


Next we note for $k=1 \rightarrow K$ that

$$
\begin{aligned}
\int_{0}^{t_{k}} \mu^{\Delta t}(t)\left\|\frac{\partial \overline{\boldsymbol{u}}}{\partial t}(\cdot, t)\right\|_{-1}^{2} \mathrm{~d} t \quad & \int_{0}^{t_{k}} \mu^{\Delta t}\left(t-\frac{\Delta t}{2}\right)\left\|\frac{\partial \boldsymbol{u}}{\partial t}(\cdot, t)\right\|_{-1}^{2} \mathrm{~d} t+\int_{t_{k}}^{t_{k+\frac{1}{2}}} \mu^{\Delta t}\left(t-\frac{\Delta t}{2}\right)\left\|\frac{\partial \boldsymbol{u}}{\partial t}(\cdot, t)\right\|_{-1}^{2} \mathrm{~d} t \\
& -\int_{0}^{t_{\frac{1}{2}}^{2}} \mu^{\Delta t}\left(t-\frac{\Delta t}{2}\right)\left\|\frac{\partial \boldsymbol{u}}{\partial t}(\cdot, t)\right\|_{-1}^{2} \mathrm{~d} t
\end{aligned}
$$

where $t_{k+\frac{1}{2}}:=\frac{1}{2}\left(t_{k}+t_{k+1}\right), k=0 \rightarrow K$, and $\mu^{\Delta t}(t):=\mu^{\Delta t}(t+\Delta t)$ for $t \in(-\Delta t, 0]$. Noting for $t \in[0, T]$ that

$$
\mu^{\Delta t}(t)+\mu^{\Delta t}\left(t-\frac{\Delta t}{2}\right)=\frac{1}{2}+\mu^{\frac{\Delta t}{2}}(t)
$$

$(2.4,2.5)$ we have that

$$
\begin{aligned}
\int_{0}^{t_{k}}\left[\mu^{\Delta t}(t)+\mu^{\Delta t}\left(t-\frac{\Delta t}{2}\right)\right] & \left\|\frac{\partial \boldsymbol{u}}{\partial t}(\cdot, t)\right\|_{-1}^{2} \mathrm{~d} t \\
& =\int_{0}^{t_{k}}\left\|\frac{\partial \boldsymbol{u}}{\partial t}\right\|_{-1}^{2} \mathrm{~d} t+\sum_{m=1}^{2 k} \int_{\frac{(m-1) \Delta t}{2}}^{\frac{m \Delta t}{2}}\left[\mu^{\frac{\Delta t}{2}}(t)-\frac{1}{2}\right]\left\|\frac{\partial \boldsymbol{u}}{\partial t}(\cdot, t)\right\|_{-1}^{2} \mathrm{~d} t \\
& \leqslant \int_{0}^{t_{k}}\left\|\frac{\partial \boldsymbol{u}}{\partial t}\right\|_{-1}^{2} \mathrm{~d} t+\frac{\Delta t}{16} \sum_{m=1}^{2 k}\left[\left\|\frac{\partial \boldsymbol{u}}{\partial t}\left(\cdot, \frac{(m-1) \Delta t}{2}\right)\right\|_{-1}^{2}-\left\|\frac{\partial \boldsymbol{u}}{\partial t}\left(\cdot, \frac{m \Delta t}{2}\right)\right\|_{-1}^{2}\right]+C \Delta t \\
& \leqslant \int_{0}^{t_{k}}\left\|\frac{\partial \boldsymbol{u}}{\partial t}\right\|_{-1}^{2} \mathrm{~d} t+C \Delta t
\end{aligned}
$$

Furthermore as $\mu^{\Delta t}(t) \geqslant 1 / 2$ for $t \in\left(t_{m-1}, t_{m-\frac{1}{2}}\right], m=1 \rightarrow K$, it follows that

$$
\frac{\Delta t}{2} \sum_{m=1}^{k} \int_{t_{m-1}}^{t_{m-\frac{1}{2}}}\left\|\frac{\partial \boldsymbol{e}}{\partial t}\right\|_{-1}^{2} \mathrm{~d} t \leqslant \Delta t \int_{0}^{t_{k}} \mu^{\Delta t}\left\|\frac{\partial \boldsymbol{e}}{\partial t}\right\|_{-1}^{2} \mathrm{~d} t
$$

Similarly to (3.35), on noting that $\partial \boldsymbol{U} / \partial t$ is constant on $\left(t_{m-1}, t_{m}\right), m=1 \rightarrow K$, we have that

$$
\frac{1}{2} \sum_{m=1}^{k} \int_{t_{m-\frac{1}{2}}}^{t_{m}}\left\|\frac{\partial \boldsymbol{e}}{\partial t}\right\|_{-1}^{2} \mathrm{~d} t \leqslant \frac{1}{2} \sum_{m=1}^{k} \int_{t_{m-1}}^{t_{m-\frac{1}{2}}}\left\|\frac{\partial \overline{\boldsymbol{e}}}{\partial t}\right\|_{-1}^{2} \mathrm{~d} t \leqslant \int_{0}^{t_{k}} \mu^{\Delta t}\left\|\frac{\partial \overline{\boldsymbol{e}}}{\partial t}\right\|_{-1}^{2} \mathrm{~d} t
$$


Combining $(2.4,3.3 \mathrm{a}, 3.30-3.32,3.34-3.36)$ yields for $k=1 \rightarrow K$ that

$$
\begin{aligned}
\left\|\boldsymbol{e}\left(\cdot, t_{k}\right)\right\|_{-1}^{2}+\left\|\overline{\boldsymbol{e}}\left(\cdot, t_{k}\right)\right\|_{-1}^{2} & +\int_{0}^{t_{k}}\left[\gamma|\hat{\boldsymbol{e}}|_{1}^{2}+\frac{\Delta t}{2}\left\|\frac{\partial \boldsymbol{e}}{\partial t}\right\|_{-1}^{2}\right] \mathrm{d} t \\
\leqslant & 2\left\|\boldsymbol{u}\left(\cdot, \frac{\Delta t}{2}\right)-\boldsymbol{u}(\cdot, 0)\right\|_{-1}^{2}+2\left\|\left(I-\boldsymbol{Q}_{2}^{h}\right) \boldsymbol{u}(\cdot, 0)\right\|_{-1}^{2}+C \int_{0}^{t_{k}}\left[\|\boldsymbol{e}\|_{-1}^{2}+\|\overline{\boldsymbol{e}}\|_{-1}^{2}\right] \mathrm{d} t \\
& +\Delta t \int_{0}^{t_{k}}\left[\left[\mu^{\Delta t}(t)+\mu^{\Delta t}\left(t-\frac{\Delta t}{2}\right)\right]\left\|\frac{\partial \boldsymbol{u}}{\partial t}(\cdot, t)\right\|_{-1}^{2}-\left\|\frac{\partial \boldsymbol{U}}{\partial t}(\cdot, t)\right\|_{-1}^{2}\right] \mathrm{d} t \\
& +\Delta t \int_{t_{k}}^{t_{k+\frac{1}{2}}} \mu^{\Delta t}\left(t-\frac{\Delta t}{2}\right)\left\|\frac{\partial \boldsymbol{u}}{\partial t}(\cdot, t)\right\|_{-1}^{2} \mathrm{~d} t+C\left[h^{2}+(\Delta t)^{2}\right] \\
\leqslant & C \int_{0}^{t_{k}}\left[\|\boldsymbol{e}\|_{-1}^{2}+\|\overline{\boldsymbol{e}}\|_{-1}^{2}\right] \mathrm{d} t+\Delta t \int_{0}^{t_{k}}\left[\left\|\frac{\partial \boldsymbol{u}}{\partial t}\right\|_{-1}^{2}-\left\|\frac{\partial \boldsymbol{U}}{\partial t}\right\|_{-1}^{2}\right] \mathrm{d} t+C\left[h^{2}+(\Delta t)^{2}\right] .
\end{aligned}
$$

We now bound the second integral on the right-hand side of (3.37). Combining $(2.12,3.14)$ we have for $k=1 \rightarrow K$ that

$$
\int_{0}^{t_{k}}\left[\left\|\frac{\partial \boldsymbol{u}}{\partial t}\right\|_{-1}^{2}-\left\|\frac{\partial \boldsymbol{U}}{\partial t}\right\|_{-1}^{2}\right] \mathrm{d} t \leqslant J^{h}\left(\boldsymbol{U}^{k}\right)-J\left(\boldsymbol{u}\left(\cdot, t_{k}\right)\right)-J^{h}\left(\boldsymbol{Q}_{2}^{h} \boldsymbol{u}^{0}\right)+J\left(\boldsymbol{u}^{0}\right)+C\left[\Delta t+h^{2}\right] .
$$

From $(1.18,1.20,2.8,3.15)$ and $(\mathrm{D} 2)$ it follows that

$$
\left|J\left(\boldsymbol{u}^{0}\right)-J^{h}\left(\boldsymbol{Q}_{2}^{h} \boldsymbol{u}^{0}\right)\right| \leqslant C h .
$$

From $(1.18,1.19,2.4,2.8,3.15)$ it follows for $k=1 \rightarrow K$ that

$$
\left|J\left(\boldsymbol{u}\left(\cdot, t_{k}\right)\right)-J^{h}\left(\boldsymbol{\pi}^{h} \boldsymbol{u}\left(\cdot, t_{k}\right)\right)\right| \leqslant C h .
$$

It follows from $(1.17,1.19,1.25,1.31,1.44,1.45,2.4,2.10,3.5,3.8,3.15)$ and a Young's inequality that for $k=1 \rightarrow K$ and for all $\alpha>0$

$$
\begin{aligned}
J^{h}\left(\boldsymbol{U}^{k}\right)-J^{h}\left(\boldsymbol{\pi}^{h} \boldsymbol{u}\left(\cdot, t_{k}\right)\right) \leqslant & -\left[\gamma\left(\nabla \boldsymbol{U}^{k}, \nabla\left(\boldsymbol{e}^{h}\left(\cdot, t_{k}\right)\right)\right)-\left(A \boldsymbol{U}^{k}, \boldsymbol{e}^{h}\left(\cdot, t_{k}\right)\right)^{h}\right]+\frac{1}{2} \lambda_{A \max }\left|\boldsymbol{e}^{h}\left(\cdot, t_{k}\right)\right|_{h}^{2} \\
\leqslant & -\left(-\hat{\mathcal{G}}_{L}^{h}\left(\frac{\boldsymbol{U}^{k}-\boldsymbol{U}^{k-1}}{\Delta t}\right)+\boldsymbol{\Lambda}^{k}, \boldsymbol{e}^{h}\left(\cdot, t_{k}\right)\right)^{h}+C\left|\boldsymbol{e}^{h}\left(\cdot, t_{k}\right)\right|_{0}^{2} \\
\leqslant & C\left\|\frac{\boldsymbol{U}^{k}-\boldsymbol{U}^{k-1}}{\Delta t}\right\|_{-1}\left[\left\|(I-f) \boldsymbol{e}^{A}\left(\cdot, t_{k}\right)\right\|_{-1}+\left\|\boldsymbol{e}\left(\cdot, t_{k}\right)\right\|_{-1}\right] \\
& +C\left[\left\|\boldsymbol{\Lambda}^{k}\right\|\left|\boldsymbol{e}^{A}\left(\cdot, t_{k}\right)\right|_{0}+\left|\boldsymbol{e}\left(\cdot, t_{k}\right)\right|_{0}^{2}+\left|\boldsymbol{e}^{A}\left(\cdot, t_{k}\right)\right|_{0}^{2}\right] \\
\leqslant & \alpha(\Delta t)^{-1}\left\|\boldsymbol{e}\left(\cdot, t_{k}\right)\right\|_{-1}^{2}+C\left[\Delta t+h^{2}\right] .
\end{aligned}
$$

Combining (3.37-3.41) for $\alpha$ sufficiently small yields for $k=1 \rightarrow K$ that

$$
\left\|\boldsymbol{e}\left(\cdot, t_{k}\right)\right\|_{-1}^{2}+\left\|\overline{\boldsymbol{e}}\left(\cdot, t_{k}\right)\right\|_{-1}^{2}+\int_{0}^{t_{k}}\left[\gamma|\hat{\boldsymbol{e}}|_{1}^{2}+\frac{\Delta t}{2}\left\|\frac{\partial \boldsymbol{e}}{\partial t}\right\|_{-1}^{2}\right] \mathrm{d} t \leq C \int_{0}^{t_{k}}\left[\|\boldsymbol{e}\|_{-1}^{2}+\|\overline{\boldsymbol{e}}\|_{-1}^{2}\right] \mathrm{d} t+C\left[h^{2}+(\Delta t)^{2}\right] .
$$



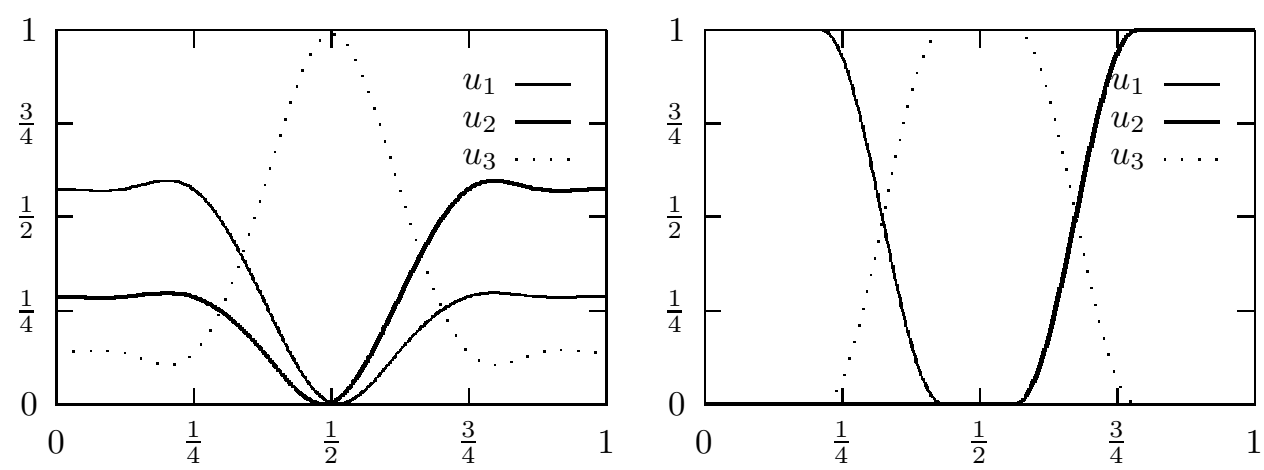

Figure 1. $\boldsymbol{u}(\cdot, t)$ for $t=0$ and 0.15 .

Next we note for $k=1 \rightarrow K$ that

$$
\begin{aligned}
\int_{0}^{t_{k}}\|\boldsymbol{e}\|_{-1}^{2} \mathrm{~d} t & \leqslant \sum_{m=1}^{k} \int_{t_{m-1}}^{t_{m}}\left[\left\|\boldsymbol{e}\left(\cdot, t_{m-1}\right)\right\|_{-1}+\left\|\int_{t_{m-1}}^{t} \frac{\partial \boldsymbol{e}}{\partial s} \mathrm{~d} s\right\|_{-1}\right]^{2} \mathrm{~d} t \\
& \leqslant 2 \Delta t \sum_{m=0}^{k-1}\left\|\boldsymbol{e}\left(\cdot, t_{m}\right)\right\|_{-1}^{2}+2 \sum_{m=1}^{k} \int_{t_{m-1}}^{t_{m}}\left(\int_{t_{m-1}}^{t}\left\|\frac{\partial \boldsymbol{e}}{\partial s}\right\|_{-1} \mathrm{~d} s\right)^{2} \mathrm{~d} t \\
& \leqslant 2 \Delta t \sum_{m=0}^{k-1}\left\|\boldsymbol{e}\left(\cdot, t_{m}\right)\right\|_{-1}^{2}+2(\Delta t)^{2} \int_{0}^{t_{k}}\left\|\frac{\partial \boldsymbol{e}}{\partial t}\right\|_{-1}^{2} \mathrm{~d} t .
\end{aligned}
$$

The desired result (3.20) then follows from combining $(3.42,3.43)$ and a similar bound with $\boldsymbol{e}$ replaced by $\overline{\boldsymbol{e}}$, applying a discrete Gronwall inequality and noting $(1.32,2.4,3.8)$.

\section{Numerical EXPERIMENT}

We chose $N=3$,

$$
\left(\begin{array}{rrr}
2 / 3 & -1 / 3 & -1 / 3 \\
-1 / 3 & 2 / 3 & -1 / 3 \\
-1 / 3 & -1 / 3 & 2 / 3
\end{array}\right) \quad \text { and } \quad A=-\left(\begin{array}{lll}
0 & 1 & 1 \\
1 & 0 & 1 \\
1 & 1 & 0
\end{array}\right)
$$

We note that the eigenvalues of $L$ and $A$ are respectively $0,1,1$ and $-2,1,1$.

As no exact time dependent solution to $(\mathrm{P})$ is known with a free boundary, a comparison between the solutions of $\left(\mathrm{P}^{h, \Delta t}\right)$ on a coarse mesh, $\boldsymbol{U}$, with that on a fine mesh, $\boldsymbol{u}$, was made. The data used in the experiment on the coarse meshes were $\Omega \equiv(0,1), \gamma=0.005, T=0.15, \Delta t=0.16 h$ and $h=1 /(M-1)$ where $M=2^{p}+1$ $(p=5,6,7,8)$. The data were the same for the fine mesh except that $M=2^{11}+1$.

As $\lambda_{A \max }=1$ and $\|L\|=1$ the condition in Theorem 3.3 on $\Delta t$ is that $\Delta t<4 \gamma=0.02$. The initial data $\boldsymbol{u}^{0}$ was taken to be the clamped (complete) cubic spline with $u_{1}^{0}$ taking the values $\{s, s, s, s / 2, s / 128, s / 4, s / 2, s / 2, s / 2\}$ at the equally spaced points $i / 8, i=0 \rightarrow 8 ; u_{2}^{0}(x)=u_{1}^{0}(1-x)$ and $u_{3}^{0}(x)=1-u_{1}^{0}(x)-u_{2}^{0}(x)$. In the above we chose $s=1024 / 1779$, so that $f u_{n}^{0} \approx 1 / 3, n=1 \rightarrow 3$; see Figure 1 , where we plot $\boldsymbol{u}(\cdot, 0)$ and $\boldsymbol{u}(\cdot, 0.15)$. Note that $\boldsymbol{u}^{0} \in \boldsymbol{H}^{3}(\Omega) \backslash \boldsymbol{H}^{4}(\Omega), \partial \boldsymbol{u}^{0} / \partial \boldsymbol{\nu}=\mathbf{0}$ and $\boldsymbol{u}^{0} \geq \delta \mathbf{1}$ for $\delta=1.04 \times 10^{-3}$. Hence $\boldsymbol{u}^{0}$ satisfies the assumptions (D2). This choice of initial data also ensured that there was a free boundary for $\boldsymbol{U}^{1}$ on all of the coarse meshes. In addition for all choices of $h$, the discrete initial data $\boldsymbol{Q}_{2}^{h} \boldsymbol{u}^{0}$ satisfied (3.3b).

We used the iterative method discussed in [2] to solve for $\boldsymbol{U}^{k}$ at each time level in $\left(\mathrm{P}^{h, \Delta t}\right)$ with the same stopping criterion: maximum difference of the successive iterates was less than $10^{-7}$. 
We computed the quantity

$$
\zeta_{n}:=\left[\frac{1}{K} \sum_{k=1}^{K}\left|\pi^{h} u_{n}(\cdot, k \Delta t)-U_{n}^{k}(\cdot)\right|_{1}^{2}\right]^{1 / 2} \quad n=1,2,3
$$

and obtained the following table of values to three significant figures:

$\begin{array}{lcccc}M & 33 & 65 & 129 & 257 \\ \zeta_{1}^{2} & 2.24 \times 10^{-3} & 3.45 \times 10^{-4} & 7.22 \times 10^{-5} & 1.70 \times 10^{-5} \\ \zeta_{2}^{2} & 2.24 \times 10^{-3} & 3.45 \times 10^{-4} & 7.22 \times 10^{-5} & 1.70 \times 10^{-5} \\ \zeta_{3}^{2} & 7.75 \times 10^{-4} & 2.27 \times 10^{-4} & 4.68 \times 10^{-5} & 1.11 \times 10^{-5} \\ \|\boldsymbol{\zeta}\|^{2} & 5.26 \times 10^{-3} & 9.18 \times 10^{-4} & 1.91 \times 10^{-4} & 4.51 \times 10^{-5}\end{array}$

We see that the ratio of consecutive $\|\boldsymbol{\zeta}\|^{2}$ is approximately 5.7, 4.8 and 4.2 which are around 4.0 , the rate of convergence proved in Theorem 3.3.

\section{REFERENCES}

[1] J.W. Barrett and J.F. Blowey, An error bound for the finite element approximation of a model for phase separation of a multi-component alloy. IMA J. Numer. Anal. 16 (1996) 257-287.

[2] J.W. Barrett and J.F. Blowey, Finite element approximation of a model for phase separation of a multi-component alloy with non-smooth free energy. Numer. Math. 77 (1997) 1-34.

[3] J.W. Barrett and J.F. Blowey, Finite element approximation of a model for phase separation of a multi-component alloy with non-smooth free energy and a concentration dependent mobility matrix. $M^{3} A S 9$ (1999) 627-663.

[4] J.F. Blowey and C.M. Elliott, The Cahn-Hilliard gradient theory for phase separation with non-smooth free energy, part II: Numerical analysis. Eur. J. Appl. Math. 3 (1992) 147-179.

[5] J. Rulla, Error analysis for implicit approximations to solutions to Cauchy problems. SIAM J. Numer. Anal. 33 (1996) 68-87. 